bodies are not universal. Global platforms like EQUATOR (Enhancing the QUAlity and Transparency Of health Research) Network help to enhance the reporting standards and credibility of research articles. New Systematic-review data-repositories will allow access to raw data for early research-comparison. 'Artificial Intelligence' software with automatic-machine-learning-algorithms can filter flawed researches from genuine ones by identifying outliers early. The rising competition between journals and media's insatiable hunger for newer scientific discoveries has laid a lot of pressure on the researchers. Media sensationalism should be resisted to avoid uncertainties and reduce pressure over scientists. Government funding agencies and Universities can pull the brakes to statistical malpractices by cutting-off support and rewards for trials with flawed structures. The cornerstones of evidence-based medicine rely on adequateappraisal, systematic-review and authenticating-evidence.

Conclusions A good clinical study must be non-biased, with a core of ethics and laced with scientific advancements. Peer-review, Refined Reporting-standards, Raw-data publication in open-access journals, Systematic-review repositories, Data-synthesis and comparison, Independent-funders, multisite studies and limiting conflicts of interests can safeguard rational research. Not all researchers or funding-bodies intend to fraud. But, too many temptations along the route incite them to indulge in scientific-misconduct which is a critical waste of funds. It is, therefore, essential to promote the agenda of 'Transparency-or-Perish' rather 'Publish-orPerish'!

\section{IN THE AGE OF GOOGLE, PATIENTS ARE TAKING ON MORE ACTIVE ROLES IN THEIR MEDICAL CARE, AND THE PHYSICIAN'S WORD IS NO LONGER SEEN AS GOSPEL}

Victoria McKinnon. MMaster University, Hamilton, Canada

\subsection{6/bmjebm-2019-EBMLive.109}

As a result, the medical community has witnessed increasing numbers of patients turn from Western medicine towards alternative healthcare. While some professionals scoff at this shift, in many ways it is the logical result of exposing a nonmedical community to the overwhelming, and, at times, misleading, body of evidence that supports Western medical practices. One particularly egregious example of this shift is the rise of anti-vaccination sentiment. Many anti-vaxxer parents have invested their time in understanding the 'science' that backs such sentiment. This leads one to believe that it is not a decreasing societal intelligence or lack of interest that has resulted in a movement away from modern medicine; rather, the shift is more likely attributable to the accessibility of alternative medicine texts as compared to scientific research. Medical research at best makes sense to the medical community; at worst, only those with niche training in the topic understand it. As such, how can we expect findings to translate over adequately to the patient community? If we wish to make research relevant to patients, and if we hope to ensure physicians with limited statistical backgrounds are applying new research appropriately, then it is required that we develop a system whereby an outside party conveys study results clearly, succinctly, and with minimised bias. I propose that this is best accomplished through the widespread integration of lay summaries alongside study abstracts. A lay summary is essentially a précis that has been written in layperson's terms, allowing those without a background in the subject to obtain an accurate understanding of it. Lay summaries are already used in the context of grant committees, as patient opinion is considered a critical component of clinical research funding. They have also been shown to benefit medical professionals on the committees with differing areas of expertise. The summary itself should be written by an outside body, ideally a statistician, who can maintain some level of objectivity and reliability in the reporting of results. Another benefit of using an outside reviewer is that it holds the authors accountable to the robustness of their methods; knowing that someone with an expertise in statistical analysis will be reviewing the study and summarising the results adds a certain level of expectation and pressure for the analysis to be performed well. As with abstracts, the lay summary should be made publicly available. Thus, even if patients do not have access to the study, they can still obtain a fundamental understanding of the available evidence and its statistical strength. The major barrier for this idea is the cost of the statistician. However, I would argue that the addition of lay summaries could broaden a journal's readership and strengthen its reputation as a reputable source of knowledge, thereby redeeming any monetary losses while simultaneously increasing accessibility, reducing bias, and minimising questionable research practices. 